\title{
Public Abstract
}

First Name: Zhilin

Middle Name:

Last Name: Liu

Degree: PhD

Academic Program: Animal Science

Adviser's First Name: Eric

Adviser's Last Name: Antoniou

Co-Adviser's First Name:

Co-Adviser's Last Name:

Graduation Term: Fall

Graduation Year: 2006

Title: Gene Expression Profiling of Bovine Ovarian Follicular Selection

Cows must get pregnant within three months after parturition to maintain a twelve-month calving interval. Lack of, or abnormal ovarian follicular development will delay the timely reestablishment of pregnancy and is a major factor associated with reduced reproductive efficiency. It is estimated that the cost for prolonged calving interval is about \$25 per cow per month. Understanding follicular development, especially the mechanisms of follicular selection, would help in the identification of cows with healthy follicular waves and increase reproductive efficiency. We have studied gene expression profiles of ovarian follicles around the stage of selection and identified many differentially expressed genes, such as immune related and cell cycle related genes. These findings provide insights into the molecular mechanisms regulating ovarian follicular selection and could lead to the development of better management tools to increase cow reproductive efficiency. 